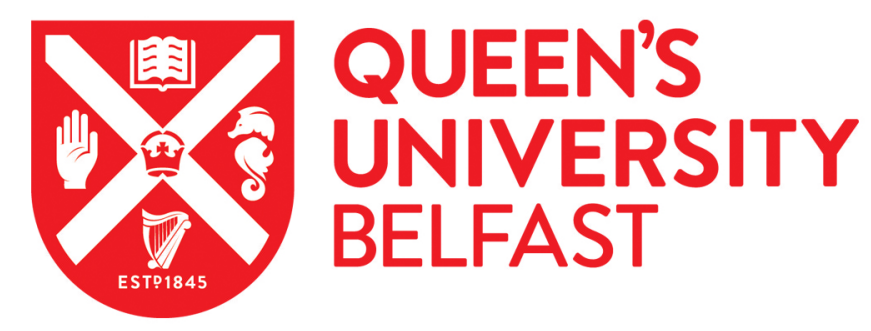

\title{
Partial Relay Selection and Energy Detection for MCIM based Dual- Hop Multiple DF Relay Network
}

Crawford, J., \& Ko, Y. (2017). Partial Relay Selection and Energy Detection for MCIM based Dual-Hop Multiple DF Relay Network. In IEEE 18th International Worksop on Signal Processing Advances in Wireless Communications, SPAWC 2017 (pp. 1-5). Institute of Electrical and Electronics Engineers Inc.. https://doi.org/10.1109/SPAWC.2017.8227648

Published in:

IEEE 18th International Worksop on Signal Processing Advances in Wireless Communications, SPAWC 2017

\section{Document Version:}

Peer reviewed version

Queen's University Belfast - Research Portal:

Link to publication record in Queen's University Belfast Research Portal

Publisher rights

(C) IEEE. This work is made available online in accordance with the publisher's policies. Please refer to any applicable terms of use of the publisher.

\section{General rights}

Copyright for the publications made accessible via the Queen's University Belfast Research Portal is retained by the author(s) and / or other copyright owners and it is a condition of accessing these publications that users recognise and abide by the legal requirements associated with these rights.

Take down policy

The Research Portal is Queen's institutional repository that provides access to Queen's research output. Every effort has been made to ensure that content in the Research Portal does not infringe any person's rights, or applicable UK laws. If you discover content in the Research Portal that you believe breaches copyright or violates any law, please contact openaccess@qub.ac.uk. 


\title{
Partial Relay Selection and Energy Detection for MCIM based Dual-Hop Multiple DF Relay Network
}

\author{
James Crawford, Youngwook Ko \\ Institute of Electronics, Communications and Information Technology \\ Queens University of Belfast \\ Belfast, NI, BT3 9DT, United Kingdom \\ Email: \{jcrawford23, y.ko\}@qub.ac.uk
}

\begin{abstract}
This paper analyzes the performance of multicarrier index modulation (MCIM) with greedy detection (GD) in a dual hop, multiple decode and forward (DF) relay network. We propose a novel partial relay selection (PRS) technique combined with index modulation and its energy detection. For performance analysis, we derive novel closed form solutions for both the average pairwise error probability (PEP) and the average symbol error probability (SEP) at the relays and destination over Nakagami- $m$ fading channels. Unlike the information theoretical works, with presence of positive detection error in the relays, the derived expressions provide a useful insight into the error performance of PRS based MCIM in a multiple DF relay network under various fading conditions. Also, we address the impacts of both relays and different rates of sub-carrier index activation.
\end{abstract}

\section{INTRODUCTION}

Recently the demand for lower power consumption, and lower complexity, with increased reliability has increased significantly in machine type communications (MTC) applications. For this, 3GPP has released the standardization of the MTC (e.g., 3GPP's NB-M2M [1]), aiming to provide a low data-rate, high reliability solution. In recent years there has been a growing interest in index modulation techniques. In particular, multicarrier index modulation (MCIM) uses indices of sub-carriers as an additional degree of freedom which results in the transmission of additional data without consuming additional power or bandwidth. MCIM has been considered as a promising candidate to improve the reliability in various designs of MTC.

Improvements to the performance of MCIM have been presented in [2-4]. In [5], multiple-input multiple-output (MIMO) MCIM (or MIMO-OFDM-IM) was introduced, and later [6] looked at MCIM as a promising candidate for $5 \mathrm{G}$ mobile broadband as it provided better bit error rate (BER) performance, a flexible system design, and a better compatibility to large MIMO setups. However, there has been less of a focus on the development of MCIM for device-to-device (D2D) systems, where system complexity is critical. In order to address this issue, [7] proposed a low complexity energy based detection scheme for MCIM. In [8], the performance of MCIM was analyzed for in-vehicular D2D wireless networks. The inferior power gain of the energy based detection scheme was improved with diversity combining in [9] .

Relay technology is an efficient solution that improves the reliability of a communication network without much expenditure. In [10], an overview of relay selection for mutlicarrier systems is presented. In [11], multicarrier relay selection schemes for OFDM are presented. However, to the best of the authors knowledge partial relay selection (PRS) for multicarrier systems has not been investigated. For PRS, channel information is only required locally at the source node, which enables to reduce overall complexity at high selection diversity.

In this work, we consider a MCIM based multiple DF relay network, where a source device intends to communicate with a destination device via multiple relays and a direct link. For each transmission, we propose the MCIM with greedy detection (GD) leveraging benefits of designing a novel partial relay selection (PRS) scheme. The main contributions can be summarized as follows; we create an MCIM structure with a novel partial relay selection scheme and low complexity $\mathrm{GD}$; for the performance evaluation, we derive closed form solutions for both the average pairwise error probability (PEP) and the average symbol error probability (SEP) of the relays and destination over Nakagami- $m$ fading channels; with presence of positive detection errors in relays and destination, the derived expressions provide a useful insight into the error performance of PRS based MCIM in multiple DF relay networks as well as the impact of PRS and relays, under various fading conditions.

\section{SySTEM MODEL}

\section{A. Multi-relayed MCIM}

Consider a MCIM based multiple DF relay communication network where a source node $(S)$ intends to communicate to the destination $(D)$, via $Q$ DF relays $\left(R_{r}\right)$, where $r=\{1, \ldots, Q\}$ as illustrated in Fig. 1. Assume that the transmission between $S \rightarrow R_{r}, S \rightarrow D$, and $R_{r} \rightarrow D$, occur in two orthogonal time slots. In the first time slot the transmission between $S \rightarrow R_{r}$ and $S \rightarrow D$ occur. For each transmission link, we employ a MCIM that has $N_{c}=G N$ total sub-carriers, being divided into $G$ clusters of $N$ subcarriers. For MCIM, $K$ of $N$ sub-carriers per cluster are dynamically activated according to the incoming bit-stream. Hence, $K$ activated sub-carriers are modulated with $M$-ary symbols, while the remaining $N-K$ sub-carriers are zero padded. 
The set of $K$ active sub-carrier indices is denoted by $\mathbf{J}_{g}=\left[i_{g}(1), \ldots, i_{g}(K)\right]$, for cluster $g$, where $i_{g}(k) \in\{(g-1) N+1, \ldots, g N\}, g=1, \ldots, G$, and $k=1, \ldots, K$. Correspondingly, a cluster of $K M$ ary symbols is denoted as $\mathbf{s}_{g}=\left[s_{g}(1), \ldots, s_{g}(K)\right]$ where $s_{g}(k) \in \mathcal{S}$ is a $M$-ary symbol and $\mathcal{S}$ denotes the complex symbol constellation. Each MCIM signal per cluster is generated using both $\mathbf{J}_{g}$ and $\mathbf{s}_{g}$, as $\mathbf{x}_{g}=$ $\left[x_{g}((g-1) N+1), x_{g}((g-1) N+2), \cdots, x_{g}(g N)\right]^{T}$, where $x_{g}(\alpha) \in \mathcal{S}$, for $\alpha \in \mathbf{J}_{g}$, and $x_{g}(\widetilde{\alpha})=0$, for $\widetilde{\alpha} \notin \mathbf{J}_{g}$. The transmit power of non-zero data symbols is defined as $\mathbb{E}\left\{\left|x_{g}(\alpha)\right|^{2}\right\}=N / K \cdot E_{s}$, where $E_{s}$ is the average power per $M$-ary symbol and $\omega=N / K$ is the power allocation coefficient. Subsequently, $G$ clusters are concatenated together to create the MCIM block, i.e., $\mathbf{x}=\left[\mathbf{x}_{1}^{T}, \mathbf{x}_{2}^{T}, \cdots, \mathbf{x}_{G}^{T}\right]^{T}$.

The total number of active sub-carrier index combinations is $\left(\begin{array}{l}N \\ K\end{array}\right)$, which is the binomial coefficient of $N$ choose $K$. For simplicity and efficient mapping of data bits, $C=2^{\left\lfloor\log _{2}\left(\begin{array}{l}N \\ K\end{array}\right)\right\rfloor}$ combinations are used. Hence, in each transmission per cluster, $m_{J, g}=\left\lfloor\log _{2}\left(\begin{array}{l}N \\ K\end{array}\right)\right\rfloor$ bits are used to modulate the combination of active sub-carrier indices and $m_{S, g}=K \log _{2} M$ bits determine the $M$-ary symbols to modulate the sub-carriers of the active indices.

Suppose that the CFR between $S \rightarrow R_{r}, S \rightarrow D$, and $R_{r} \rightarrow D$, can be defined, in the frequency domain, as $\mathbf{H}_{\Delta}=$ $\operatorname{diag}\left(h_{\Delta}(1), \ldots, h_{\Delta}\left(N_{c}\right)\right)$, where $\Delta \in\left\{S R_{r}, S D, R_{r} D\right\}$, and $h_{\Delta}(\alpha)$ are assumed to follow flat Nakagami- $m$ fading distribution for $\alpha \in \mathbf{J}_{g}, \forall g$, being identically distributed (i.i.d.), where $m \geq 0.5$ is the fading parameter which represents the fading severity.

In the first time slot, the received signals in the frequency domain can be given as,

$$
\mathbf{y}_{S \Lambda}=\sqrt{P_{S}} \mathbf{H}_{S \Lambda} \mathbf{x}+\mathbf{n}_{S \Lambda},
$$

where $\Lambda \in\left\{R_{r}, D\right\}, P_{S}$ is the transmit power at $S, \mathbf{x}$ is an $N \times 1$ MCIM block, and $\mathbf{n}_{S \Lambda}=\left[n_{S \Lambda}(1), \ldots, n_{S \Lambda}\left(N_{c}\right)\right]^{T}$ is an independent additive complex Gaussian noise vector between $S$ and $\Lambda$, and $\mathbf{n}_{S \Lambda} \sim \mathcal{C N}\left(\mathbf{0}, \mathbf{I} N_{0}\right)$, where $\mathbf{I}$ is the identity matrix and $N_{0}$ is noise variance. The average SNR per active sub-carrier is defined as $\bar{\gamma}=E_{s} / \tau N_{0}$, where $\tau=1 / \omega$ is the sub-carrier sparsity ratio.

In the second time slot the received signals from $R_{r} \rightarrow D$ and $S \rightarrow D$ transmission are combined using MRC. Where, the received signal from $R_{r} \rightarrow D$ is given as,

$$
\mathbf{y}_{R_{r} D}=\sqrt{P_{R_{r}}} \mathbf{H}_{R_{r} D} \widetilde{\mathbf{x}}+\mathbf{n}_{R_{r} D}
$$

where $P_{R}$ is the transmit power at $R_{r}$, and $\widetilde{\mathbf{x}}$ is the imperfectly decoded $N \times 1$ MCIM block that was recovered at $R_{r}$. In addition, $\mathbf{y}_{S R_{r}}$ and $\mathbf{y}_{R_{r} D}$ are combined at $D$ using maximal ratio combining (MRC). Hence, the SNR of the $\alpha^{\text {th }}$ active sub-carrier at $D$ is defined as,

$$
\gamma_{D, \alpha}=\frac{P_{S}\left|h_{S D}(\alpha)\right|^{2}}{N_{0}}+\frac{P_{R}\left|h_{R_{r} D}(\alpha)\right|^{2}}{N_{0}},
$$

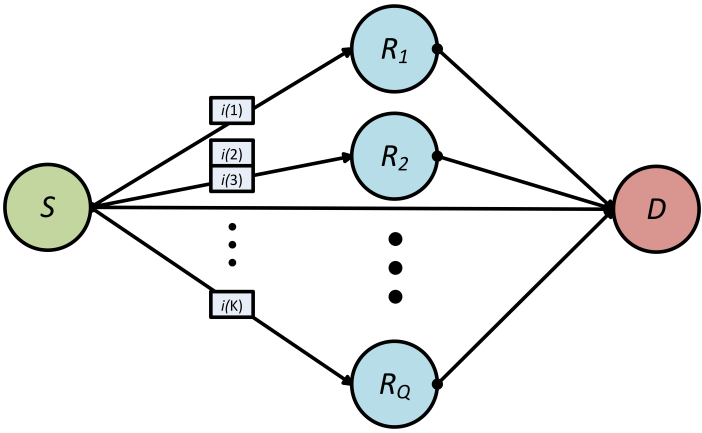

Fig. 1. Dual hop multiply DF relay network employing MCIM with GD and PRS

For simplicity in analysis and without loss of generality, we propose the partial relay detection process in terms of only one cluster and the cluster index $g$ is omitted from subscripts hereafter as each cluster operates independently.

\section{B. Opportunistic Partial Relay Selection (OPRS)}

Opportunistic partial relay selection for MCIM sub-carriers (OPRS) chooses the relay(s) that provides the largest channel gain, per active sub-carrier, between $S$ and relays, $\mathbf{R} \in$ $\left\{R_{1}, \ldots, R_{r}, \ldots, R_{Q}\right\}$. Suppose that perfect channel state information (CSI) of $\mathbf{H}_{S R}=\left[\mathbf{H}_{S R_{1}}, \ldots, \mathbf{H}_{S R_{Q}}\right]^{T}$ is available at $S$. The criterion for the relay selection at the $\alpha^{\text {th }}$ active sub-carrier is to find the best among $Q$ relays, whose channel gain satisfies,

$$
\tilde{r}_{\alpha}=\arg \max _{r \in\{1, \ldots, Q\}}\left|h_{S r}(\alpha)\right|^{2},
$$

where $\tilde{r}_{\alpha}$ denotes the index of the selected relay for the $\alpha^{\text {th }}$ active sub-carrier. Notice that each active sub-carrier will be relayed by the selected relay $\tilde{r}_{\alpha}$ and this per-sub-carrier process allows at most $K$ relays to be chosen for relaying.

Let $\mathcal{R} \in\left\{\tilde{r}_{1}, \ldots, \tilde{r}_{K}\right\}$ denote the set of indices of selected relays, where $|\mathcal{R}| \leq K$ due to the possibility that $\tilde{r}_{i}=\tilde{r}_{j}$. Based on the OPRS selection rule, the SNR of the $\alpha^{\text {th }}$ active sub-carrier at the selected relay $\tilde{r}_{\alpha}$ is defined as,

$$
\gamma_{S \tilde{r}_{\alpha}}(\alpha)=\frac{P_{S}\left|h_{S \tilde{r}_{\alpha}}(\alpha)\right|^{2}}{N_{0}} .
$$

\section{Greedy Detector (GD) Receiver}

In the first time slot GD is used at $\tilde{r}_{\alpha}$ to recover the data sent from $S$, while the second time slot GD is used at $D$ to recover the combined data sent from $S$ and $\tilde{r}_{\alpha}$. The GD requires a two stage detection process, where the indices of the active sub-carriers and the corresponding $M$-ary symbols are estimated separately, and $\leq K$ active sub-carrier indices and data symbols are recovered at $\tilde{r}_{\alpha}$. In the first stage, GD measures the combined-output signal energy of each subcarrier $\left|y_{\Delta}(j)\right|^{2}$, where $j \in\{\alpha, \tilde{\alpha}\}$, and detects $K$ active sub-carriers with the greatest energy, which is given as,

$$
\hat{\alpha}=\arg \max _{j}\left|y_{\Delta}(j)\right|^{2} .
$$


In the second stage, the non-zero $M$-ary symbols in $\mathbf{x}$ and $\tilde{\mathbf{x}}$ are detected, applying the maximum likelihood (ML) decision individually to the $K$ estimated active sub-carriers, which is given as,

$$
z(\hat{\alpha})=\arg \min _{x(\hat{\alpha}) \in \mathcal{S}}\left|y_{\Delta}(\hat{\alpha})-h(\hat{\alpha}) x(\hat{\alpha})\right|^{2},
$$

where $z(\hat{\alpha}) \in\{\hat{x}(\hat{\alpha}), \hat{\widetilde{x}}(\hat{\alpha})\}$ are the detected elements from $\mathbf{x}$ and $\tilde{\mathbf{x}}$ respectively, and $h(\hat{\alpha})$ is the $\alpha^{\text {th }}$ diagonal element of $\mathbf{H}_{\Delta}$. Further details about the GD process is found in [9].

\section{ERROR PROBABILITY ANALYSIS}

\section{A. Pairwise Error Probability (PEP) Analysis}

1) Instantaneous PEP for OPRS-MCIM with GD: The incorrect detection of sub-carrier indices can occur at $D$ under two different scenarios. Firstly, an error occurs at $D$ but not at $\mathcal{R}$, i.e., $P_{1}=\left(1-P E P_{\mathcal{R}}\right) \times P E P_{D}$, where $\left(1-P E P_{\mathcal{R}}\right)$ is the complementary PEP at $\mathcal{R}$, and $P E P_{D}$ is the PEP at $D$. Secondly, when an error occurs at $\mathcal{R}$, i.e., $P_{2}=P E P_{\mathcal{R}}$, where $P E P_{\mathcal{R}}$ is the PEP at $\mathcal{R}$. Hence, the overall instantaneous PEP can be determined by a miss-detection of sub-carrier indices. Using the law of the total probability, this can be represented as,

$$
P_{P E P}=\frac{K}{N} \sum_{\alpha=1}^{N} P E P_{\mathcal{R}}+P E P_{D}-P E P_{\mathcal{R}} P E P_{D},
$$

The approximate instantaneous PEPs at $\Lambda \in\left\{\tilde{r}_{\alpha}, D_{\alpha}\right\}$ is formulated as [8],

$$
P E P_{\Lambda} \approx \frac{N-K}{2} e^{-\frac{\gamma_{\Lambda}}{2}}
$$

Substituting (9) into (8) yields our instantaneous PEP expression, which is given as,

$$
\begin{aligned}
P_{P E P} & \approx \frac{K}{N} \sum_{\alpha=1}^{N}\left\{\frac{N-K}{2} e^{-\frac{\gamma_{S \tilde{r}_{\alpha}}}{2}}+e^{-\frac{\gamma_{D, \alpha}}{2}}\right. \\
& \left.-\frac{N-K}{2} e^{-\frac{\gamma_{S \tilde{r}_{\alpha}+\gamma_{D, \alpha}}}{2}}\right\},
\end{aligned}
$$

2) Average PEP for OPRS-MCIM with GD: The approximate average PEP can be found by taking the expectation of the (10) over the SNRs, which is given as,

$$
\begin{aligned}
\bar{P}_{P E P} & =K \underbrace{\mathbb{E}_{\gamma_{S \tilde{r}_{\alpha}}}\left\{P E P_{\mathcal{R}}\right\}}_{I_{1}}+K \underbrace{\mathbb{E}_{\gamma_{D, \alpha}}\left\{P E P_{D}\right\}}_{I_{2}} \\
& -K \underbrace{\underbrace{}_{\gamma_{S \tilde{r}_{\alpha}}}\left\{P E P_{\mathcal{R}}\right\}}_{I_{1}} \underbrace{\mathbb{E}_{\gamma_{D, \alpha}}\left\{P E P_{D}\right\}}_{I_{2}} .
\end{aligned}
$$

Using the order statistics probability density function (PDF) of $\gamma_{S \tilde{r}_{\alpha}}$ becomes mathematically intractable for more complex generalized channels models, i.e., Nakagami- $m$. Hence, we use the extreme value theory approach which enables an asymptotic distribution for the maximum of $Q$ i.i.d. RVs. This approach is shown to provide a tight bound to the exact PDF for as little as $Q=10$, [12]. Given $\gamma_{S \tilde{r}_{\alpha}}$ is Gamma distributed with shape parameter $m$, and rate parameter $m / \bar{\gamma}$, the asymptotic distribution of $Q$ Gamma random variables can be given in the form of a Gumbel random variable, i.e., $z=\left(\gamma_{S \tilde{r}_{\alpha}}-\mu_{Q}\right) / \beta_{Q}$, where $\mu_{Q}$ and $\beta_{Q}$ are the generalized inverse and the auxiliary function, respectively, for the Gamma distribution [12], which are given as $\mu_{Q}=\bar{\gamma} / m P^{-1}(m,(1-1 / Q))$, and $\beta_{Q}=$ $\Gamma(m)\left[1-P\left(m, \mu_{Q} m / \bar{\gamma}\right)\right] /\left[(m / \bar{\gamma})^{m} \mu_{Q}^{m-1} e^{-\mu_{Q} m / \bar{\gamma}}\right]$, where $P(m, x)=1 / \Gamma(m) \int_{0}^{x} t^{m-1} e^{-t} \mathrm{~d} t$ is the regularized lower incomplete Gamma function, $\Gamma(m)$ is the gamma function, and $P^{-1}($,$) is the inverse regularized lower incomplete Gamma$ function, which does not have a closed form solution but is available in mathematical software packages, such as Matlab, and Mathematica.

Hence, the Gumbel PDF is given as,

$$
g_{z}(z)=\frac{\exp \left[-z-e^{-z}\right]}{\beta_{Q}}
$$

The combined SNR at the output of the MRC at $D$ is the sum of two i.i.d. Gamma RV, where the PDF is given as,

$$
f_{\gamma_{D, \alpha}}\left(\gamma_{D, \alpha}\right)=\frac{1}{\Gamma(2 m)}\left(\frac{m}{\bar{\gamma}}\right)^{2 m} \gamma_{D, \alpha}^{2 m-1} e^{-\frac{\gamma_{D, \alpha} m}{\bar{\gamma}}} .
$$

Firstly, (12) and $\gamma_{S \tilde{r}_{\alpha}}=z \beta_{Q}+\mu_{Q}$ are substituted into $I_{1}$ from (11),

$$
I_{1}=\frac{(N-K) e^{-\frac{\mu_{Q}}{2}}}{2 \beta_{Q}} \int_{-\frac{\mu_{Q}}{\beta_{Q}}}^{\infty} \exp \left[-\left(\frac{\beta_{Q}}{2}+1\right) z-e^{-z}\right] \mathrm{d} z .
$$

From (14), an additional substitution, $z=t-\frac{\mu_{Q}}{\beta_{Q}}$ is made, which yields,

$I_{1}=\frac{(N-K) e^{-\frac{\mu_{Q}}{2}}}{2 \beta_{Q}} \int_{0}^{\infty} \exp \left[-\left(\frac{\beta_{Q}}{2}+1\right) t-\left(e^{\frac{\mu_{Q}}{\beta_{Q}}}\right) e^{-t}\right] \mathrm{d} t$,

Using $[13, \quad(3.331-1)]$, we can obtain $I_{1}=$ $N-K / 2 \beta_{Q} e^{-\mu_{Q} / 2} \gamma\left(\frac{\beta_{Q}}{2}+1, e^{-\mu_{Q} / \beta_{Q}}\right)$, where $\gamma(A, B)$ is the lower incomplete Gamma function.

Secondly, substituting (13) into $I_{2}$ yields,

$$
I_{2}=\frac{(N-K)(m / \bar{\gamma})^{2 m}}{2 \Gamma(2 m)} \int_{0}^{\infty} \gamma_{D, \alpha}^{2 m-1} e^{-\gamma_{D, \alpha} \frac{2 m+\bar{\gamma}}{2 \bar{\gamma}}} \mathrm{d} \gamma_{D, \alpha} .
$$

Referring to [13, (3.381-4)], (16) can be obtained in closed form as, $I_{2}=N-K / 2(2 m / 2 m+\bar{\gamma})^{2 m}$.

Hence, using the above closed-form outcomes back into (11) yields the average PEP expression, which is given as,

$$
\begin{aligned}
\bar{P}_{P E P} & =K \frac{N-K}{2}\left\{\frac{e^{-\frac{\mu_{Q}}{2}}}{\beta_{Q}} \gamma\left(\frac{\beta_{Q}}{2}+1, e^{\frac{\mu_{Q}}{\beta_{Q}}}\right)\right. \\
& +\left(\frac{2 m}{2 m+\bar{\gamma}}\right)^{2 m}-\frac{N-K}{2 \beta_{Q}} e^{-\frac{\mu_{Q}}{2}} \times \\
& \left.\gamma\left(\frac{\beta_{Q}}{2}+1, e^{\frac{\mu_{Q}}{\beta_{Q}}}\right)\left(\frac{2 m}{2 m+\bar{\gamma}}\right)^{2 m}\right\} .
\end{aligned}
$$




\section{B. Symbol Error Probability (SEP) Analysis}

1) Instantaneous SEP for OPRS-MCIM with GD: Similar to (8), the overall instantaneous SEP is formulated as,

$$
P_{\text {sym }}=\frac{K}{N} \sum_{\alpha=1}^{N} P_{\mathcal{R}}+P_{D}-P_{\mathcal{R}} P_{D},
$$

and the instantaneous SEP at $\Lambda \in\left\{\tilde{r}_{\alpha}, D_{\alpha}\right\}$ is given as,

$$
P_{\Lambda}=P E P_{\Lambda}+P_{Q A M, \Lambda}-\left[P E P_{\Lambda} \times P_{Q A M, \Lambda}\right],
$$

where $P_{Q A M, \Lambda}$ is the instantaneous SEP of QAM symbols, per active sub-carrier, which is approximated as [14],

$$
P_{Q A M, \Lambda} \approx \frac{1}{6} e^{-\frac{3 \gamma_{\Lambda}}{2 M-2}}+\frac{1}{2} e^{-\frac{4 \gamma_{\Lambda}}{2 M-2}} .
$$

Substituting (20) and (9), into (19), yields,

$$
\begin{aligned}
P_{\Lambda} & \approx \frac{N-K}{2} e^{-\frac{\gamma_{\Lambda}}{2}}+\frac{1}{6} e^{-\frac{3 \gamma_{\Lambda}}{2 M-2}}+\frac{1}{2} e^{-\frac{\gamma_{\Lambda}}{2 M-2}} \\
& -\frac{N-K}{12} e^{-\frac{\gamma_{\Lambda}(M+2)}{2 M-2}}-\frac{N-K}{4} e^{-\frac{\gamma_{\Lambda}(M+3)}{2 M-2}}
\end{aligned}
$$

Hence, the instantaneous SEP is found by substituting (21) into (18). However, for brevity the solution has been left out.

2) Average SEP for OPRS-MCIM with GD: Here, the solution to the average SEP is obtained by taking the expectation of the instantaneous SEP over the SNRs, which is given as,

$$
\begin{aligned}
\bar{P}_{\text {sym }} & =K \underbrace{\mathbb{E}_{\gamma_{S \tilde{r}_{\alpha}}}\left\{P_{\mathcal{R}}\right\}}_{I_{A}}+K \underbrace{\mathbb{E}_{\gamma_{D, \alpha}}\left\{P_{D}\right\}}_{I_{B}} \\
& -K \underbrace{\mathbb{E}_{\gamma_{S_{\tilde{r}}}}\left\{P_{\mathcal{R}}\right\}}_{I_{A}} \underbrace{\mathbb{E}_{\gamma_{D, \alpha}}\left\{P_{D}\right\}}_{I_{B}} .
\end{aligned}
$$

Provided that (21) is the sum of exponentials, $I_{A}$ can be broken down into a sum integrals. All integrals in $I_{A}$ are found to be in the same form, i.e., $\int_{0}^{\infty} \exp \left(-A x-B e^{-x}\right) d x$. Hence, using [13, (3.331-1)] and after some manipulations, $I_{A}$ yields (23), found on the next.

Similarly, $I_{B}$ can be broken down into a sum integrals, which are found to be in the same form, i.e., $\int_{0}^{\infty} x^{\nu-1} e^{-\mu x} d x$. Referring to [13, (3.381-4)], $I_{B}$ yields,

$$
\begin{aligned}
I_{B} & =\frac{N-K}{2}\left(\frac{2 m}{2 m+\bar{\gamma}}\right)^{2 m} \\
& +\frac{1}{6}\left(\frac{2 m(M-1)}{2 m(M-1)+3 \bar{\gamma}}\right)^{2 m}+\frac{1}{2}\left(\frac{m(M-1)}{m(M-1)+2 \bar{\gamma}}\right)^{2 m} \\
& -\frac{N-K}{4}\left\{\frac{1}{3}\left(\frac{2 m(M-1)}{2 m(M-1)+\bar{\gamma}(M+2)}\right)^{2 m}\right. \\
& \left.+\left(\frac{2 m(M-1)}{2 m(M-1)+\bar{\gamma}(M+3)}\right)^{2 m}\right\} .
\end{aligned}
$$

Finally, the average SEP can be found easily by inserting (23) and (24) into (22), whose expression is omitted for simplicity.

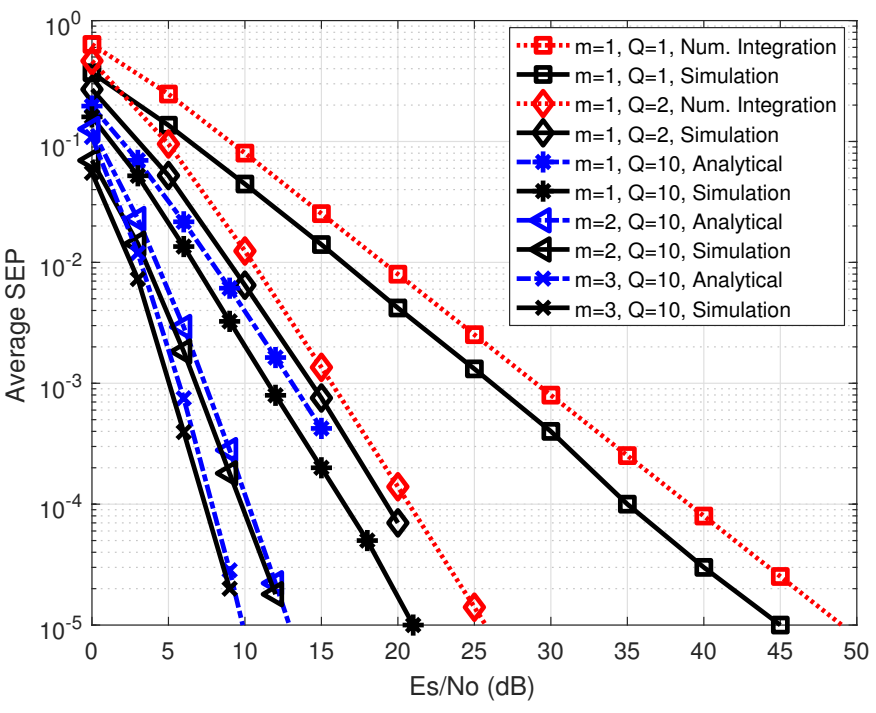

Fig. 2. Average SEP performance of OPRS-MCIM with GD for $N=4$, $K=1, M=4, m=\{1,2,3\}, Q=\{1,2,10\}$, and data-rate $=4$ bits per cluster

\section{Numerical Results AND Discussion}

In this section, numerical and simulation results for a dual hop OPRS-MCIM with GD are presented. The MCIM system with $N_{c}=128$ total sub-carriers has various configurations of $G, N, K, M$, and $Q$. In particular $N=4, K \leq 2, M=4$, $m \in\{1,2,3\}$, and $Q=\{1,2,10\}$. Here, the average SEP is evaluated on a per cluster basis, as each cluster of $N$ subcarriers is detected individually.

Fig. 2 depicts the average SEP performance of the OPRSMCIM with GD, with $N=4, K=1, M=4, m=\{1,2,3\}$, and $Q=\{1,2,10\}$. It is observed that the average SEP performance is significantly improved as $Q$ is increased. For example, the power gain of $12 \mathrm{~dB}$ can be achieved for the average SEP of $10^{-3}$ between $Q=1$ and $Q=2$. However, between $Q=2$ and $Q=10$ the power gain is only marginal. This can be explained by the average SEP being dominated by the weakest hop. Interestingly, despite MRC at the receiver, a diversity order of 1 is observed at the destination. Intuitively, this is explained by the imperfect decoding at the relays. The results in red depict (22), which has been numerically integrated using the exact PDF of $\gamma_{S \tilde{r}_{\alpha}}$, as the asymptotic solution does not hold for lower values of $Q$. The analytical and numerically integrated results provide a tight bound to the simulation results with a $2 \mathrm{~dB}$ gap for the average SEP of $10^{-3}$ when $Q=m=1$, where the gap decreases when $Q$ and $m$ are increased.

In Fig. 3, the average SEP performance of a dual hop OPRSMCIM with GD, is also analyzed, with $N=4, K=2$, $M=4, m=\{1,2,3\}$, and $Q=10$. It is observed that as $m$ is increased the average SEP significantly decreases. In particular, the power gain of $4 \mathrm{~dB}$ is achieved for the average SEP of $10^{-3}$. The analytical results provide a tight bound to the simulation for the average SEP $\leq 10^{-1}$. In particular, there is as $2 \mathrm{~dB}$ gap between analytical and simulation results for 


$$
\begin{aligned}
I_{A}= & \frac{N-K}{2 \beta_{Q}} e^{-\frac{\mu_{Q}}{2}} \gamma\left(\frac{\beta_{Q}}{2}+1, e^{\frac{\mu_{Q}}{\beta_{Q}}}\right)+\frac{e^{-\frac{3 \mu_{Q}}{2 M-2}}}{6 \beta_{Q}} \gamma\left(\frac{3 \beta_{Q}}{2 M-2}+1, e^{\frac{\mu_{Q}}{\beta_{Q}}}\right)+\frac{e^{-\frac{4 \mu_{Q}}{2 M-2}}}{2 \beta_{Q}} \gamma\left(\frac{4 \beta_{Q}}{2 M-2}+1, e^{\frac{\mu_{Q}}{\beta_{Q}}}\right) \\
& -\frac{N-K}{12 \beta_{Q}} e^{-\frac{\mu_{Q}(M+2)}{2 M-2}} \gamma\left(\frac{\beta_{Q}(M+2)}{2 M-2}+1, e^{\frac{\mu_{Q}}{\beta_{Q}}}\right)-\frac{N-K}{4 \beta_{Q}} e^{-\frac{\mu_{Q}(M+3)}{2 M-2}} \gamma\left(\frac{\beta_{Q}(M+3)}{2 M-2}+1, e^{\frac{\mu_{Q}}{\beta_{Q}}}\right),
\end{aligned}
$$

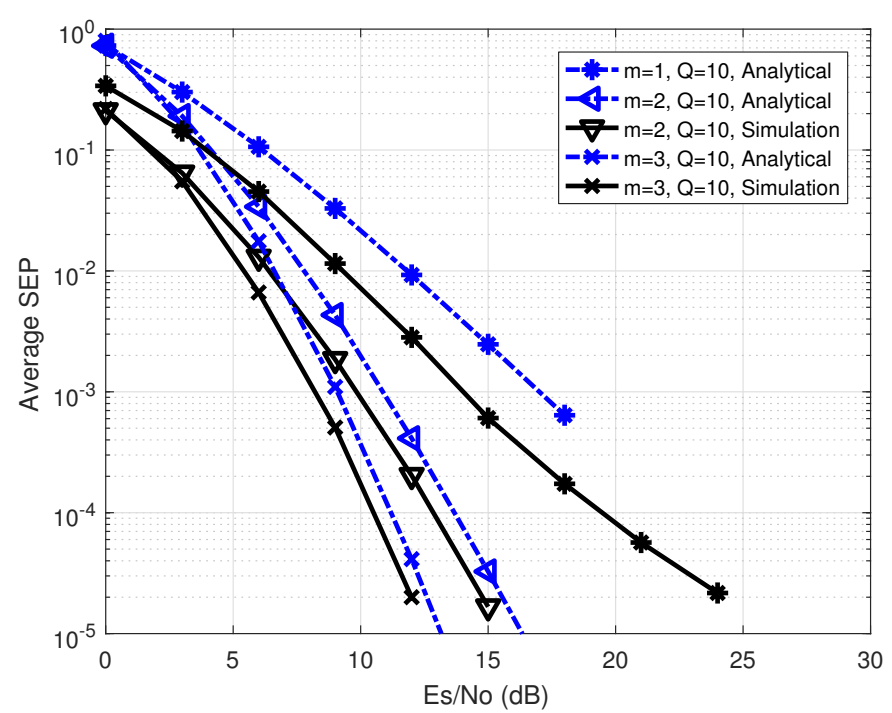

Fig. 3. Average SEP performance of OPRS-MCIM with GD for $N=4$, $K=2, M=4, m=\{1,2,3\}, Q=10$, and data-rate $=6$ bits per cluster

the average SEP of $10^{-3}$ when $m=1$. Moreover, in Fig. 3 the approximate expression becomes tighter for large $m(\geq 2)$, providing less than $1 \mathrm{~dB}$ difference between simulations and analytical results.

\section{CONCLUSIONS}

We proposed the new partial relay selection scheme for the dual hop multiple DF relay network. Taking into account the benefits of both the MCIM and the PRS, we designed the best relay selection rule that has decreased the error probability. For the performance evaluation, closed form expressions for both the average PEP and the average SEP have been derived over a general Nakagami- $m$ fading channels. The derived expressions provide a useful insight into the error performance of hybrid MCIM and OPRS under various fading conditions. In presence of detection errors in the relays, we clearly showed that the achievable diversity order is limited to two while the power gains is proportional to the number of relays and active subcarrier index rates.

\section{REFERENCES}

[1] "Celluar system support for ultra-low complexity and low throughput internet of things (CIoT)," 3GPP, TR 45.820 V13.1.0, Nov. 2015.

[2] E. Basar, U. Aygolu, E. Panayirci, and H. V. Poor, "Orthogonal frequency division multiplexing with index modulation," IEEE Trans. on Signal Process., vol. 61, no. 22, pp. 5536-5549, Nov. 2013.

[3] Y. Xiao, S. Wang, L. Dan, X. Lei, P. Yang, and W. Xiang, "OFDM with interleaved subcarrier-index modulation," IEEE Commun. Lett., vol. 18, no. 8, pp. 1447-1450, Aug. 2014.

[4] R. Fan, Y. Yu, and Y. L. Guan, "Generalization of orthogonal frequency division multiplexing with index modulation," IEEE Transactions on Wireless Commun., vol. 14, no. 10, pp. 5350-5359, Oct. 2015.

[5] E. Basar, "Multiple-input multiple-Output OFDM with index modulation," IEEE Signal Process. Lett., vol. 22, no. 12, pp. 2259-2263, Dec. 2015.

[6] — " "On multiple-input multiple-Output OFDM with index modulation for next generation wireless networks," IEEE Trans. Signal Process., vol. 64, no. 15, pp. 38683878, Aug. 2016.

[7] J. Crawford and Y. Ko, "Low complexity greedy detection method with generalized multicarrier index keying OFDM," Proc. IEEE Pers., Indoor., Mobile Radio Commun., pp. 688-693, Aug. 2015.

[8] E. Chatziantoniou, J. Crawford, and Y. Ko, "Performance analysis of a low complexity detector for MCIK-OFDM over TWDP fading," IEEE Commun. Lett., vol. 20, pp. 1251-1254, Jun. 2016.

[9] — " "A low complexity detector with MRC diversity reception for MCIK-OFDM," Proc. IEEE Pers., Indoor., Mobile Radio Commun., 2016.

[10] P. Guo, Y. Bai, M. Ma, S. Wu, and S. Dang, "Relay technology for multi-carrier systems: a research overview," Computer, communication, control, and information technology (C3IT), pp. 1-5, Feb 2015.

[11] S. Dang, J. Coon, and D. E. Simmons, "Combined bulk/per-subcarrier relay selection in two-hop OFDM systems," Vehicul. Technol. Conf. (VTC), May 2016.

[12] P. Embrechts, C. Kluppelberg, and T. Mikosch, Modelling Extremal Events. Springer, 1996.

[13] I. Gradshteyn and I. Ryzhik, Table of Integrals, Series, and Products, A. Jeffrey and D. Zwillinger, Eds. Academic Press, 2007.

[14] A. Suraweera, H. and J. Armstrong, "Simple and accurate approximation to the SEP of rectangular QAM in arbitrary nakagami-m fading channels," IEEE Commun. Lett., vol. 11, no. 5, pp. 426-428, May 2007. 